
cos e bioquímicos. Horticultura Brasileira 25: 527-532.

\title{
Estimativa da diversidade genética de germoplasma de cebola via descritores morfológicos, agronômicos e bioquímicos
}

\author{
Anne Giselle R Buzar ${ }^{1}$; Valter R Oliveira ${ }^{2}$; Leonardo S Boiteux ${ }^{2,3}$ \\ ${ }^{1}$ Universidade de Brasília, FAV, Campus Universitário Darcy Ribeiro, 70910-900 Brasília-DF; ${ }^{2}$ Embrapa Hortaliças, C. postal 218, \\ 70359-970 Brasília-DF; ${ }^{3}$ Bolsista CNPq; annebuzar@yahoo.com.br; valter@cnph.embrapa.br; boiteux@cnph.embrapa.br
}

\section{RESUMO}

A divergência genética de 64 acessos de cebola (Allium cepa L.) tropicais e subtropicais do banco de germoplasma da Embrapa Hortaliças foi avaliada utilizando 23 descritores morfológicos, bioquímicos e agronômicos. A distância generalizada de Mahalanobis $\left(\mathrm{D}^{2}\right)$ foi usada como medida de dissimilaridade e os métodos de otimização de Tocher e hierárquico do Vizinho mais Próximo foram empregados para o agrupamento dos acessos. O germoplasma de cebola avaliado apresentou base genética relativamente ampla. As cultivares EX 3000 e Régia foram os acessos geneticamente mais similares. A maior distância genética estimada foi entre os acessos VAL 14 e Beta Cristal. O método de otimização de Tocher proporcionou a formação de treze grupos, enquanto o procedimento hierárquico do vizinho mais próximo possibilitou a formação de doze grupos (corte com $43 \%$ de dissimilaridade) A maioria dos acessos dos tipos Grano e Granex, agronomicamente considerados de dias curtos e com ciclo de maturação precoce, se concentraram em mesmo grupo de similaridade genética. À exceção do acesso Tampico White, os demais acessos de bulbos brancos do tipo indústria (Beta Cristal; Dehydrator \# 8; Dehydrator \# 6; Primero e White Creole) exibiram estreito relacionamento. Os acessos TX 24, Baia Periforme Super Precoce, EX 3001, Excel, Red Creole, H 688, Franciscana IPA 10 e Roxa IPA 3 exibiram alta dissimilaridade genética. Os caracteres teor de açúcares totais, comprimento de bulbo, número de dias para a colheita, porcentagem de bulbos com diâmetro entre 70 e $90 \mathrm{~mm}$ e massa média de bulbos foram responsáveis por $58 \%$ de toda a variabilidade genética observada entre os acessos. O estudo da divergência entre acessos é importante subsídio na escolha de parentais divergentes e complementares a fim de desenvolver populações segregantes com ampla base genética em programas de melhoramento genético de cebolas tropicais.

Palavras-chave: Allium cepa L., distância genética, cebola tropical, cebola subtropical, análise de agrupamento, germoplasma.

\section{ABSTRACT}

Estimating genetic diversity of onion germplasm via morphological, agronomic, and biochemical descriptors

The genetic diversity of 64 tropical and subtropical onion accessions (Allium cepa L.) belonging to the Embrapa Hortaliças collection was estimated using 23 morphological, agronomic, and biochemical descriptors. Methods of multivariate statistical analysis (Mahalanobis' $\mathrm{D}^{2}$ distances, Tocher's cluster analysis and nearestneighbor method) were employed in order to obtain the average genetic distances among accessions and to perform the clustering. The onion germplasm displayed a relatively large genetic base. Cultivars EX 3000 and Régia were the two most similar accessions. The highest mean genetic distance was observed between cultivars VAL 14 and Beta Cristal. The Tocher's optimization method revealed the formation of 13 groups, whereas the nearest-neighbor method revealed the formation of 12 groups (cutting point of $43 \%$ of dissimilarity. The majority of the accessions belonging to Grano and Granex cultivars (agronomically classified as short-day and early-maturing types) clustered together in the same group. The white bulb accessions for processing (Beta Cristal; Dehydrator \# 8; Dehydrator \# 6; Primero and White Creole), with the exception of Tampico White, displayed a small genetic divergence. The accessions TX 24, Baia Periforme Super Precoce, EX 3001, Excel, Red Creole, H 688, Franciscana IPA 10 and Roxa IPA 3 displayed a large genetic divergence. The descriptors total sugar content, bulb length, number of days from planting to harvesting (i.e. plant cycle), percentage of bulbs with transversal diameter between 70 and $90 \mathrm{~mm}$, and average bulb weight were responsible for $58 \%$ of the genetic variability present among accessions. Studies of divergence among accessions are important to choose divergent parentals but complementary genetic materials would allow the development of useful segregating populations with wide genetic basis for tropical onion breeding programs.

Keywords: Allium cepa L., genetic distance, tropical onion, subtropical onion, grouping analysis, germplasm.

\section{(Recebido para publicação em 11 de julho de 2006; aceito em 15 de outubro de 2007)}

$\mathrm{O}$ estabelecimento do germoplasma brasileiro de cebola amarela (Allium cepa L.) coincidiu com a chegada de imigrantes portugueses e açorianos que colonizaram as regiões de Rio Grande e Pelotas, no estado do Rio Grande do Sul, no início do século XIX (França \& Candeia, 1997). A partir de acessos de cebola introduzidos da Europa foram selecionadas as populações Baia Periforme no Rio Grande do Sul e Crioula, em Santa Catarina. As cultiva- res do grupo Baia Periforme apresentam características de ampla adaptação a diferentes condições de cultivo no Brasil, nível alto de tolerância a doenças e boa conservação pós-colheita. As cultivares do grupo Crioula são mais adaptadas à região Sul, possuem bulbos de coloração amarela-escura, de ótima conservação pós-colheita e ampla aceitação no mercado. Cultivares importadas dos grupos varietais Grano e Granex são tradicionalmente cultivadas no Nor- deste e principalmente no Sudeste do Brasil (França \& Candeia, 1997; Melo \& Boiteux, 2001; Costa et al., 2002). Nos estados do Nordeste e nas capitais das regiões Centro-Oeste e Sudeste, especialmente em Belo Horizonte, existe também elevada demanda por cultivares de bulbos arroxeados e pungentes.

As primeiras cultivares híbridas de cebola disponibilizadas no Brasil foram as do grupo Granex. Estes híbridos foram desenvolvidos a partir de 1950, nos 
Estados Unidos da América, cruzandose linhagens do tipo Grano com linhagens tolerantes ao fungo Pyrenochaeta terrestris, extraídas da cultivar Bermuda (Currah, 2002). Diversos híbridos do tipo Granex tornaram-se populares no Brasil, incluindo Granex 33, Granex 429, Mercedes, Princesa e Superex (Melo \& Boiteux, 2001). Cultivares do tipo Granex possuem alto potencial produtivo, precocidade de maturação, resistência ao pendoamento, sabor, odor e pungência suaves, além de resistência à doença raiz rosada (Costa et al., 2002). São, no entanto, mais suscetíveis aos fungos Alternaria porri e Colletotrichum gloeosporioides que as cultivares nacionais e, a exemplo das cultivares Grano, são facilmente danificadas pelo manuseio demasiado, resultando em curta conservação pós-colheita, mesmo sob condições de temperaturas amenas (Currah \& Green, 1997).

Um marco na história recente do melhoramento genético e que contribuiu para o incremento da diversidade genética do germoplasma brasileiro de cebola, foi a criação do programa de pesquisa da Empresa Pernambucana de Pesquisa Agropecuária, em 1972. Este programa resultou na geração de diversas cultivares do tipo Baia Periforme, da chamada série IPA, que foram selecionadas para adaptação a condições tropicais (latitudes $8-9^{\circ}$ ), permitindo a bulbificação em dias mais curtos e com temperaturas médias mais elevadas que as observadas na região Sul. Nas décadas de 1970 e 1980, outros programas de melhoramento genético públicos e privados foram implementados para atender às demandas das diferentes regiões produtoras. Um dos destaques regionais tem sido as ações do programa da Estação Experimental da Empresa de Pesquisa Agropecuária e Extensão Rural de Santa Catarina, em Ituporanga, iniciadas em 1976, cujas atividades de melhoramento concentram-se no germoplasma do tipo Crioula. $\mathrm{Na}$ Embrapa, os trabalhos de melhoramento genético de cebola foram iniciados em 1977. Os programas da Embrapa visam disponibilizar cultivares de cebola adaptadas às mais variadas condições edafoclimáticas, sistemas de cultivo e preferências regionais, tendo sido disponibilizadas até o presente sete cul- tivares: Conquista, São Paulo, Alfa Tropical e Beta Cristal pela Embrapa Hortaliças; Aurora, Primavera e BRS Cascata pela Embrapa Clima Temperado e BRS Alfa São Francisco pela Embrapa Semi-Árido (Pereira et al., 2000; Costa et al., 2005).

Os parâmetros utilizados para quantificar a variabilidade genética em germoplasma vegetal têm sido as estimativas de divergência entre e dentro de populações e, ou acessos. Estes estudos fornecem importantes subsídios para o conhecimento da variabilidade dos acessos e possibilitam melhor gerenciamento de bancos de germoplasma, facilitando a identificação de genitores adequados para a formação de populações com ampla base genética (Cruz et al., 2003; Sudré et al., 2005). Além disso, a expressão da heterose em híbridos depende da existência de divergência genética entre os acessos parentais (Falconer, 1981). Devido à associação estreita entre divergência genética e heterose, estas análises podem ser úteis para a predição de cruzamentos que otimizem a obtenção de combinações híbridas mais produtivas e vigorosas (Melchinger, 1999). Neste contexto, a identificação de genitores divergentes e já adaptados a determinadas condições ambientais constitui-se em fator importante para o sucesso comercial de cultivares híbridas (Dias \& Kageyama, 1991; Silva, 1996; Spaner et al., 1996; Abreu, 1997).

A utilização de descritores morfológicos de caráter métrico tem sido um procedimento generalizado na caracterização da variabilidade de germoplasma, inclusive de cebola (D’Ennequin et al., 1997). Quando a caracterização é realizada por meio de dados morfológicos, a quantificação da diversidade entre acessos só terá significado se a divergência fenotípica refletir a divergência genética (Dias \& Kageyama, 1991). Vários procedimentos estatísticos multivariados encontram-se disponíveis para análise de divergência genética e para a seleção de descritores mais relevantes. O estudo de divergência feito por análise de agrupamento tem a finalidade de reunir, por algum critério de classificação, os progenitores em vários grupos, de tal modo que haja maior homogeneidade dentro do grupo e maiores níveis de heterogeneidade entre os grupos (Cruz et al., 2003).

Diferentes populações e, ou acessos do germoplasma brasileiro de cebolas apresentam ampla variabilidade fenotípica quanto ao formato, firmeza, tamanho, cor e qualidades organolépticas dos bulbos, entre outras características. No entanto, poucos estudos têm sido implementados catalogando esta diversidade e estimando a divergência genética entre acessos que compõem este germoplasma. $\mathrm{Na}$ Embrapa Clima Temperado foram caracterizados 24 acessos incluindo cultivares locais e comerciais de cebola (Barbieri et al., 2005). Na Embrapa Hortaliças, Valencio et al. (2004) caracterizaram 22 acessos de cebola pertencentes ao grupo 'Grano' com base em descritores morfológicos. O presente trabalho teve como objetivo ampliar a caracterização morfológica, agronômica e bioquímica da coleção de cebolas tropicais e subtropicais pertencentes à Embrapa Hortaliças e utilizar estas características na estimativa da diversidade genética deste germoplasma.

\section{MATERIAL E MÉTODOS}

Sessenta e quatro acessos (Tabela 1 e Figura 1) do banco de germoplasma de cebola da Embrapa Hortaliças foram cultivados em campo, na área experimental da Embrapa Hortaliças, localizado em Brasília, a $15^{\circ} 56^{\prime} 00^{\prime \prime} \mathrm{S}$ e $48^{\circ}$ 06’ 00" W, altitude de $998 \mathrm{~m}$. O clima local segundo a classificação de KÖPPEN é do tipo Cwa, isto é, clima subtropical de altitude, com verão chuvoso, inverno seco, temperatura do mês mais quente superior a $23^{\circ} \mathrm{C}$ e temperatura do mês mais frio inferior a $13^{\circ} \mathrm{C}$. O experimento foi instalado em área com Latossolo Vermelho Amarelo. O semeio foi realizado em 25/04/04, o transplante em 28/06/04 e a colheita, entre 28/08 e 18/10/04. As mudas, produzidas em sementeira em campo, foram transplantadas com três a quatro folhas definitivas e cultivadas segundo as práticas culturais recomendadas para a cultura (Oliveira \& Boiteux, 2004). O delineamento experimental utilizado foi blocos 
completos casualizados com três repetições (50 plantas por repetição). As parcelas foram de $1,4 \mathrm{~m}^{2}$, compostas por quatro linhas espaçadas de $35 \mathrm{~cm}$. O solo da área experimental, antes da adubação, apresentou: $\mathrm{pH}\left(\mathrm{H}_{2} \mathrm{O}\right)=5,1 ; \mathrm{P}=11$ $\mathrm{mg} \mathrm{dm}^{-3}$ (mehlich-1); M.O.= $31 \mathrm{~g} \mathrm{dm}^{-3}$; $\mathrm{K}, \mathrm{Na}, \mathrm{S}, \mathrm{Cu}, \mathrm{Fe}, \mathrm{Zn}, \mathrm{Mn}$ e B, respectivamente $=176 ; 82 ; 13 ; 0,5 ; 15,7 ; 4,1$; 26,5 e $0,6 \mathrm{mg} \mathrm{dm}^{-3}$. $\mathrm{Na}$ adubação de plantio foram utilizados 40, 300 e 160 $\mathrm{kg} \mathrm{ha}^{-1}$ de N, $\mathrm{P}_{2} \mathrm{O}_{5}$ e $\mathrm{K}_{2} \mathrm{O}$ (fórmula 4-3016), respectivamente. Foram feitas duas adubações em cobertura com $20 \mathrm{~kg}$ de $\mathrm{N}$ (sulfato de amônio) cada, a primeira 20 dias após o transplante e, a segunda, 25 dias após a primeira, tomando como base as recomendações da Emater-DF e Comissão de Fertilidade do Solo do Estado de Minas Gerais (Emater-DF, 1987; CFSEMG, 1999).

A caracterização morfológica, agronômica e bioquímica dos acessos foi feita de acordo com os descritores propostos pelo IPGRI-ECP/GR-AVRDC (2001). Os caracteres avaliados e utilizados no estudo da divergência genética foram comprimento da folha mais longa, (medida da inserção no pseudocaule até a extremidade da folha); largura máxima da folha mais longa; número de folhas; comprimento do pseudocaule; diâmetro do pseudocaule; diâmetro do bulbo; comprimento do bulbo; razão comprimento/diâmetro do bulbo; número de dias para a colheita, a partir da semeadura; porcentagem de bulbos com diâmetro transversal $>90$ $\mathrm{mm}$; porcentagem de bulbos com diâmetro transversal entre 70 e $90 \mathrm{~mm}$; porcentagem de bulbos com diâmetro transversal entre 50 e $70 \mathrm{~mm}$; porcentagem de bulbos com diâmetro transversal >50 mm; porcentagem de bulbos com diâmetro transversal entre 35 e 50 $\mathrm{mm}$; porcentagem de bulbos com diâmetro transversal $<35 \mathrm{~mm}$; produtividade de bulbos totais (g/parcela); massa média dos bulbos; porcentagem de bulbos podres; porcentagem de bulbos charutos; porcentagem de bulbos duplos; porcentagem de bulbos brotados; teor de ácido pirúvico, em $\mu \mathrm{mol} / \mathrm{g}$ massa fresca de bulbo e porcentagem de açúcares totais. Os caracteres número, comprimento e largura de folhas, comprimento e diâmetro do pseudocaule,

Tabela 1. Agrupamento dos acessos de cebola pelo método de otimização de Tocher (grouping of onion accessions through Tocher's cluster analysis). Brasília, Embrapa Hortaliças, 2004.

\begin{tabular}{cl}
\hline Grupo & Acessos \\
\hline 1 & 'EX 3000'; 'Régia'; TX05'; 'TX07'; 'Houston'; 'Texas Early Grano'; 'Granex 429'; 'IPA- \\
& 2'; 'Baia Periforme'; 'Serrana'; 'L10'; 'Primavera'; 'Henry's Special PRR'; 'TX06'; \\
& 'Aurora'; 'CNPH 6400'; 'Madrugada'; 'Pira Ouro'; 'Bola Precoce'; 'São Paulo'; 'XP \\
& 8010'; 'IPA-11'; 'Optima F1'; 'XP 3237'; 'Texas Grano 502'; 'Jubileu'; 'Petroline'; 'IPA- \\
& 6'; 'TX02'; 'TX08'; 'L12'; 'TX03'; 'L26'; 'Roxa do Barreiro'; Yellow Granex'; 'H9'; 'Alfa \\
& Tropical'; 'Tampico White'; 'XP 8418'; 'H60'; TX01'; 'IPA-9'; 'L13' \\
2 & 'Beta Cristal'; 'Dehydrator \# 8'; 'Dehydrator \# 6'; 'Primero'; White Creole' \\
3 & 'Conquista'; 'Mercedes'; 'Granex 33' \\
4 & 'Baia do Cedo'; 'Crioula Alto Vale'; 'BRS Cascata' \\
5 & VAL 14'; 'Pêra Norte' \\
6 & 'IPA-3' \\
7 & 'IPA-10' \\
8 & 'H 688' \\
9 & 'Red Creole' \\
10 & 'Excel' \\
11 & 'EX 3001' \\
13 & 'Baia Periforme Super Precoce' \\
& TX 24' \\
\hline
\end{tabular}

comprimento e diâmetro do bulbo, e porcentagem de bulbos charutos foram avaliados no dia da colheita, em dez plantas bulbificadas. Os demais caracteres foram avaliados após cura dos bulbos por 60 dias em galpão. A pungência foi medida pelo método do piruvato (Schwimmer \& Weston, 1961) e, o teor de açúcares totais, pelo método fenol sulfúrico, em três bulbos representativos de cada parcela.

A análise da divergência genética entre os acessos foi feita por meio da técnica multivariada denominada análise de agrupamento, utilizando-se os métodos de otimização de Tocher e hierárquico do vizinho mais próximo, descritos por Cruz et al. (2003). Para a aplicação destas técnicas, inicialmente procedeu-se às análises de variâncias e estimação das matrizes de médias e de covariâncias. Em seguida foram estimadas as distâncias $\mathrm{D}^{2}$ de Mahalanobis, que é uma medida de dissimilaridade entre genótipos. Para o método de Tocher, o valor máximo da distância intragrupo foi estabelecido como sendo $70 \%$ do valor máximo de $\mathrm{D}^{2}$ obtido no conjunto de menores distâncias. A contribuição relativa de cada característica para a diversidade entre os acessos foi avaliada pelo método proposto por Singh (1981), que se baseia na partição do total das estimativas das distâncias $\mathrm{D}^{2}$, considerando todos os possíveis pares de indi- víduos, nas partes devidas a cada característica. As análises foram conduzidas utilizando-se os recursos computacionais do programa GENES (Cruz, 2001).

\section{RESULTADOS E DISCUSSÃO}

A diversidade genética entre um grupo de progenitores tem sido avaliada em diferentes estudos, tendo como um dos objetivos básicos a identificação de parentais para futura obtenção de combinações híbridas de maior potencial heterótico (Cruz et al., 2003). A menor estimativa de distância genética entre os acessos de cebola $\left(\mathrm{D}^{2}=13,3\right)$ ocorreu entre os acessos EX 3000 e Régia. Ambos contêm película amarela, escamas brancas, pungência baixa e são considerados cultivares de dias curtos com ciclo de maturação precoce. A maior estimativa de $\mathrm{D}^{2}(=432,3)$ ocorreu entre os acessos VAL 14 e Beta Cristal. O acesso VAL 14 é uma tradicional cultivar Argentina, de ciclo de maturação tardio, com bulbo de película marrom escura, folhas pouco cerosas, pungência intermediária e teor de açúcares totais baixo. $\mathrm{O}$ acesso Beta Cristal é uma cultivar adequada para desidratação, produção de pasta e conserva. Possui ciclo de maturação precoce, película e escamas brancas, folhas muito cerosas, pungência intermediária e teor de açúcares totais elevado. 


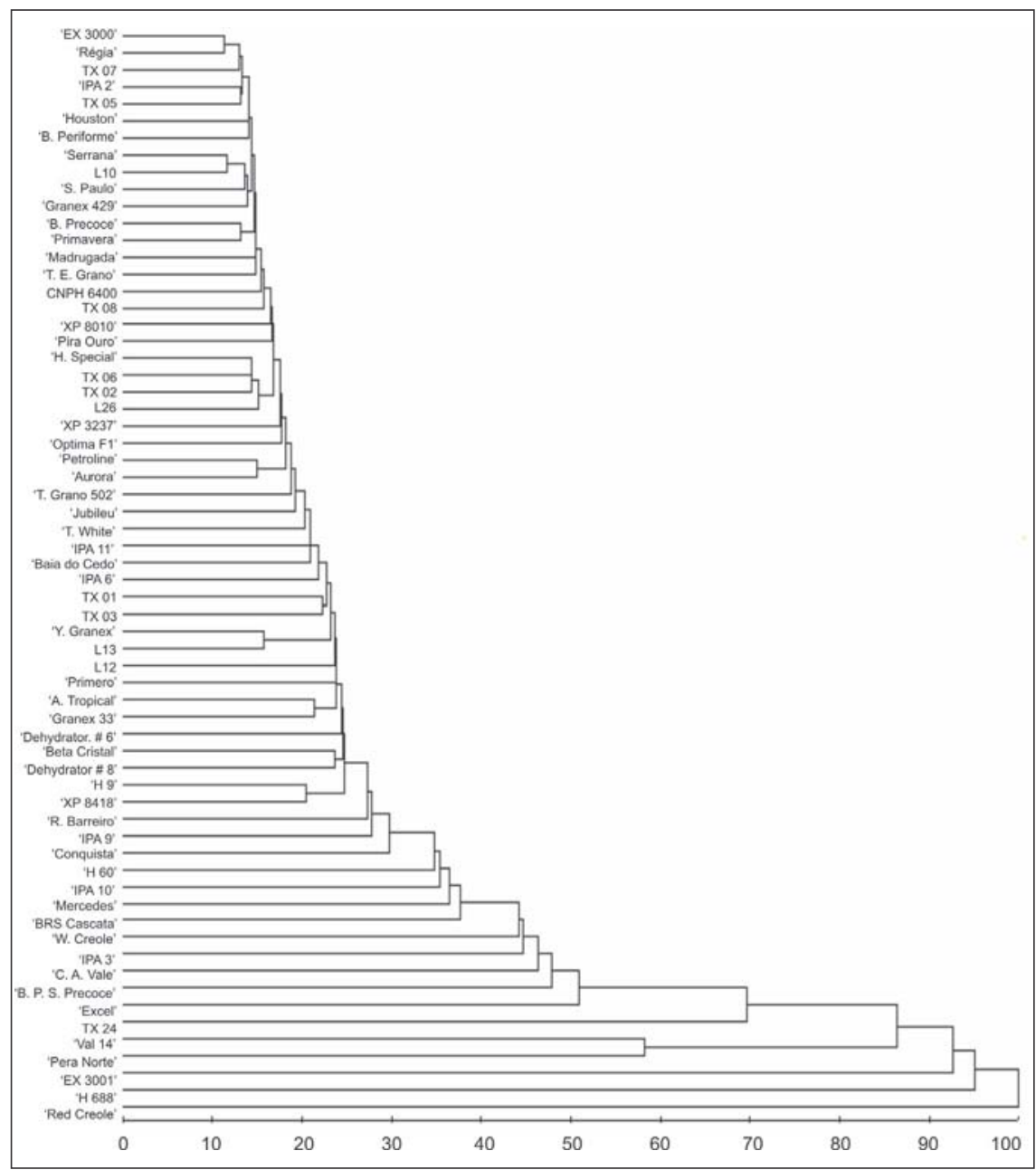

Figura 1. Dendograma de dissimilaridade genética entre acessos de cebola pelo método hierárquico do Vizinho mais Próximo". Valores na horizontal correspondem as distâncias genéticas relativas. O procedimento hierárquico do vizinho mais próximo possibilitou a identificação de doze grupos ao se realizar o corte do dendrograma considerando $43 \%$ de dissimilaridade (traço vertical) (Dendrogram of genetic dissimilarity among onion accessions through the nearest-neighbor method. Values on the horizontal line correspond to the relative genetic distance. The hierarchic method of the nearest-neighbor permitted to identify twelve groups, when cut with $43 \%$ of dissimilarity (vertical line)). Brasília, Embrapa Hortaliças, 2004.

O método de otimização de Tocher proporcionou a formação de treze grupos de acessos (Tabela 1). O procedimento hierárquico do Vizinho mais Próximo possibilitou a identificação de doze grupos, ao se realizar o corte do dendrograma considerando $43 \%$ de dissimilaridade (Figura 1), com distribuição dos acessos nos grupos muito similar ao agrupamento pelo método de Tocher.

O grupo 1 (Tabela 1) concentrou a maior parte das cultivares dos tipos Grano e Granex, agronomicamente consideradas de dias curtos e com ciclo de maturação precoce; mas englobou também cultivares de dias intermediários, colocá-los em grupos distintos. Embora a maior parte dos acessos deste grupo possuam bulbos com película variando de amarelo-claro a amarelo-escuro, acessos de película branca (Tampico White) e de película roxa (Roxa do Barreiro) também compuseram o grupo.

À exceção de 'Tampico White', os demais acessos de bulbos brancos do tipo indústria migraram para o grupo 2 , exibindo estreito relacionamento (Tabela 1). Os acessos do grupo 2 foram os que apresentaram os maiores teores de açúcares totais (de 11,6\% em Primero a $16,0 \%$ em White Creole) e todos apresentaram bulbos de formato achatado, com razão entre comprimento e diâmetro de bulbos menor que a unidade.

O grupo 3 foi composto pela cultivar Conquista, do tipo Baia Periforme e pelas cultivares Mercedes e Granex 33, do tipo Granex. As cvs. Crioula Alto Vale e BRS Cascata, desenvolvidas para a região sul do Brasil, além da cv. Baia do Cedo compuseram o grupo 4 (Tabela 1). Estes três acessos exibiram pungência elevada, ciclo do plantio a colheita bem acima da média, sendo que as cvs. Crioula Alto Vale e BRS Cascata constituem-se em genótipos importantes para o desenvolvimento de cebolas do tipo Cascuda tropicalizadas (Costa, 1995). Enquanto a cv. Baia do Cedo possui bulbos tendendo para alongado e película amarela-clara, as cvs. Crioula Alto Vale e BRS Cascata possuem, respectivamente, formato globular e de peão invertido, com película marromescura. A estreita similaridade genética exibida entre as cvs. Crioula Alto Vale e BRS Cascata está em consonância com os resultados obtidos por Barbieri et al. (2003). Em outro estudo realizado por Barbieri et al. (2005), as cultivares Aurora, Primavera, Baia Periforme, Madrugada, Crioula Alto Vale, BRS Cascata e Petroline foram classificadas dentro do mesmo grupo de similaridade. A cultivar Beta Cristal foi separada em outro grupo, resultado este também em consonância com o observado no presente estudo.

As cultivares VAL 14 e Pêra Norte, mesmo apresentando grande diferença quanto a pungência (8,7 e 13,0 $\mu \mathrm{mol}$ de ácido pirúvico/g de massa fresca, respectivamente), exibiram similaridade 
para os demais caracteres, o que resultou na alocação de ambos no grupo 5 (Tabela 1). Estas duas cultivares foram as mais tardias entre todos os acessos avaliados, com ciclo de 193 dias da semeadura a colheita.

Os acessos Roxa IPA 3, Franciscana IPA 10, H 688, Red Creole, Excel, EX 3001, Baia Periforme Super Precoce e TX 24 foram separados em grupos individuais, grupos $6,7,8,9,10,11,12 \mathrm{e}$ 13 , respectivamente (Tabela 1). Estes acessos exibiram diferenças fenotípicas acentuadas para teor de açúcares totais, que variou de 6,1\% em EX 3001 a 10,8\% em IPA10; formato de bulbo, sendo o de Excel, Red Creole e IPA 10 tipicamente achatado (comprimento/ diâmetro iguais a 0,$6 ; 0,8$ e 0,8 , respectivamente) e o da cv. Roxa IPA 3 alongado (comprimento/largura igual a 1,1); porcentagem de bulbos com diâmetro entre 70 e $90 \mathrm{~mm}$, que variou de $0 \%$ nas cvs. IPA 3 e Baia Periforme Super Precoce a $69 \%$ na cv. IPA 10; e massa média dos bulbos que variou de $33 \mathrm{~g}$ em Red Creole a 190 g em EX 3001.

Os caracteres teor de açúcares totais, comprimento de bulbo, número de dias para a colheita, porcentagem de bulbos com diâmetro entre 70 e 90 mm e massa média de bulbos foram responsáveis por $58 \%$ de toda a variabilidade genética presente entre os acessos (Tabela 3). É interessante destacar que a cv. IPA 10 é derivada de seleção em população oriunda do cruzamento entre as cvs. IPA 3 e Red Creole e que, embora a cv. Roxa IPA 3 seja essencialmente derivada da cv. Roxa do Barreiro, ambas exibiram divergência genética bastante acentuada. Este fato demonstra que pode não haver uma correspondência entre o relacionamento genético entre acessos e o relacionamento esperado levando-se em consideração exclusivamente os aspectos fenotípicos.

Conclui-se que o germoplasma de cebola avaliado neste estudo e disponível na Embrapa Hortaliças apresenta uma base genética relativamente ampla. Mesmo dentro do grupo 1, que englobou $67 \%$ dos acessos avaliados, a presença de acessos fenotipicamente distintos para diversas características analisadas indica haver ainda considerável

Tabela 3. Contribuição relativa de 23 descritores fenotípicos para a divergência genética entre acessos tropicais e subtropicais de cebola, utilizando-se a metodologia de Singh (Relative contribution of 23 fenotypic descriptor for the genetic divergence among tropical and subtropical onion acdessions, using Singh's method). (1981). Brasília, Embrapa Hortaliças, 2004.

\begin{tabular}{lc}
\hline Caracteres & Contribuição (\%) \\
\hline Teor de açúcares totais & 16,11 \\
Comprimento do bulbo & 15,37 \\
Número de dias para a colheita & 10,95 \\
Porcentagem de bulbos com diâmetro entre 70 e $90 \mathrm{~mm}$ & 8,43 \\
Massa média dos bulbos & 7,20 \\
Largura máxima da folha mais longa & 6,21 \\
Diâmetro do bulbo & 4,80 \\
Teor de ácido pirúvico & 4,29 \\
Diâmetro do pseudocaule & 3,81 \\
Porcentagem de bulbos duplos & 3,37 \\
Porcentagem de bulbos brotados & 2,55 \\
Porcentagem de bulbos com diâmetro > $90 \mathrm{~mm}$ & 2,51 \\
Comprimento do pseudocaule & 2,45 \\
\hline
\end{tabular}

diversidade genética. Uma alternativa sugerida para aumentar a eficiência e competitividade do agronegócio de cebola brasileiro tem sido diversificar a oferta de tipos varietais, procurando diferenciar o produto nacional em relação ao oferecido por mercados concorrentes (Vilela et al., 2005). Neste contexto, a caracterização fenotípica e o estabelecimento da distância genética entre estes acessos constituem-se em importantes subsídios para a escolha de progenitores divergentes e complementares para o desenvolvimento de populações segregantes em programas de melhoramento genético com cebola, visando à diversificação do mercado de cultivares nacionais.

\section{AGRADECIMENTOS}

Este trabalho faz parte da dissertação de mestrado do primeiro autor dentro do Programa de Pós-Graduação em Agronomia da Universidade de Brasília (UnB), Faculdade de Agronomia e Veterinária (FAV). Os autores agradecem pela revisão do capítulo da tese que gerou este trabalho e pelas sugestões e informações fornecidas pela Dra. Daniela Lopes Leite (Embrapa Clima Temperado). Os autores também agradecem a revisão crítica do manuscrito feita pelos Professores Dr. Jean Kleber Abreu Matos e Dr. José Ricardo Peixoto, ambos da UnB.

\section{REFERÊNCIAS}

ABREU AFB. 1997. Predição do potencial genético de populações segregantes do feijoeiro utilizando genitores inter-raciais. Lavras: Universidade Federal de Lavras, 79p. (Tese doutorado).

BARBIERI RL; LEITE DL; CHOER E; SINIGAGLIA C. 2005. Divergência genética entre populações de cebola com base em marcadores morfológicos. Ciência Rural 35: 303-306.

BARBIERI RL; LEITE DL; CHOER E; SINIGAGLIA C; MARIOT P. 2003. Similaridade genética entre acessos do banco ativo de germoplasma de cebola. In: CONGRESSO BRASILEIRO DE MELHORAMENTO DE PLANTAS, 2., Porto Seguro. Anais... Porto Seguro: SBMP, CD-ROM.

COMISSÃO DE FERTILIDADE DE SOLO DO ESTADO DE MINAS GERAIS 1999. Recomendações para o uso de corretivos e fertilizantes em Minas Gerais: $5^{\text {a }}$ Aproximação. Viçosa: Editora, 359 p.

COSTA CP. 1995. Cebola cascuda: um desafio para a cebolicultura brasileira. SOB Informa 14: 13-14.

COSTA ND; LEITE DL; SANTOS CAF; CANDEIA JA; VIDIGAL SM. 2002. Cultivares de cebola. Informe Agropecuário 23: 2027.

COSTA ND; SANTOS CAF; QUEIROZ MA; ARAÚJO HM; OLIVEIRA VR; MENDONÇA JL; CANDEIA JA. 2005. Alfa São Francisco: variedade de cebola para cultivo de verão, Brasil. Horticultura Brasileira 23. 2005. Suplemento. CD-ROM.

CRUZ CD. 2001. Programa genes (versão Windows): aplicativo computacional em genética e estatística. Viçosa: UFV, 648 p.

CRUZCD; CARNEIRO PCS; REGAZZIAJ. 2003. Modelos Biométricos Aplicados ao Melhoramento Genético. Viçosa: UFV, v. 2, 585 p. 
CURRAH L. 2002. Onions in the Tropics cultivars and country reports. In: HD RABINOWITCH \& L CURRAH (eds.). Allium Crop Science: Recent Advances. Reino Unido: CAB International Wallingford, $\mathrm{p}$. 379-407.

CURRAH L.; GREEN SM. 1997. International collaborative trials to evaluate short-day onions. Acta Horticulturae 433: 197-206.

D'ENNEQUIN MT; PANAUD O; ROBERT T; RICROCH A. 1997. Assessment of genetic relationships among sexual and asexual forms of Allium cepa using morphological traits and RAPD markers. Heredity 78: 403-409.

DIAS LAS; KAGEYAMA PV. 1991. Variação genética em espécies arbóreas e conseqüências para o melhoramento florestal. Agrotópica 3: $119-127$.

EMATER-DF. 1987. Recomendações para o uso de corretivos, matéria orgânica e fertilizantes para hortaliças: Distrito Federal. $1^{a}$ Aproximação: Brasília, DF. EMATER-DF/ EMBRAPA - CNPH, 50 p.

FALCONER DS. 1981. Introdução à Genética Quantitativa. Tradução de MA SILVA \& JC SILVA. Viçosa: UFV, Impr. Univ., 279 p.

FRANÇA JGE; CANDEIA JA. 1997. Development of short-day yellow onion for tropical environments of the Brazilian northeast. Acta Horticulturae 433: 285-287.
IPGRI, ECP/GR, AVRDC. 2001. Descriptors for Allium (Allium ssp.): International Plant Genetic Resources Institute, Rome, European Cooperative for Crop Genetic Resources Networks, Asian Vegetable Research and Development Center, 43p.

MELCHINGER AE. Genetic diversity and heterosis. In: JG COORS \& S PANDEY (eds.). Genetics and exploitation of heterosis in crops. Wisconsin: Crop Science of America, 1999. p.99-118.

MELO PCT; BOITEUX LS. 2001. Análise retrospectiva do melhoramento genético de cebola (Allium cepa L.) no Brasil e potencial aplicação de novas estratégias biotecnológicas. In: $\mathrm{CON}$ GRESSO BRASILEIRO DE MELHORAMENTO DE PLANTAS 1, Goiânia. CD ROM.

OLIVEIRA VR; BOITEUX LS. 2004.Cultivo da Cebola. Brasília, DF: Embrapa Hortaliças, (Sistema de Produção). Disponível em <http:/ /ww w.cnph.embrapa.br/paginas/ sistemas_producao/cultivo_da_cebola.htm $>$. Acesso em: 19 dez. 2006.

PEREIRA W; VIEIRA JV; MENDONÇA JL. 2000. Relatório do Workshop sobre cebolicultura no Brasil. Brasília, DF: Embrapa Hortaliças, 76 p. (Embrapa Hortaliças, Documentos 25).

SCHWIMMER S; WESTON WJ. 1961. Enzymatic development of pyruvic acid in onion as a measure of pungency. Journal of Agricultural and Food Chemistry 4: 303-304.
SILVA RM. 1996. Estudo do sistema reprodutivo e divergência genética em cupuaçuzeiro (Theobroma grandiforum Wild. Ex Spreng.) Schum. 151p. (Tese), Escola Superior de Agricultura Luiz de Queiroz, Universidade de São Paulo, Piracicaba.

SINGH D. 1981. The relative importance of characters affecting genetic divergence. Indian. Journal of Genetic and Plant Breeding 41: 237-245.

SPANER D; BRATHWAITE RAI; MATHER DE. 1996. Diallel study of open-pollinated maize varieties in Trinidad. Euphytica 90: 65-72.

SUDRÉ CP; RODRIGUES R; RIVA EM; KARASAWA M; AMARAL JÚNIOR AT. 2005. Divergência genética entre acessos de pimenta e pimentão utilizando técnicas multivariadas. Horticultura Brasileira, Brasília 23: 22-27.

VALENCIO AGRB; OLIVEIRA VR; LOPES JF; BOITEUX LS; FONSECA MEN. 2004. Estabelecimento de um banco de caracteres botânicos e agronômicos de populações de cebola suaves/doces. Horticultura Brasileira, Brasília 22. Suplemento. CD-ROM.

VILELA NJ; MAKISHIMA N; OLIVEIRA VR; COSTA ND; MADAIL JCM; CAMARGO FILHO WP; BOEING G; MELO PCT. 2005. Desafios e oportunidades para o agronegócio da cebola no Brasil. Horticultura Brasileira Brasília 23: 1029-1033. 